\title{
$\beta$-Arrestins promote podocyte injury by inhibition of autophagy in diabetic nephropathy
}

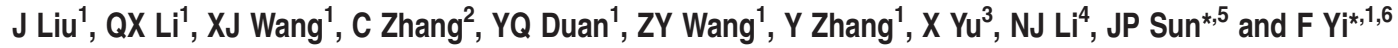

$\beta$-Arrestins are multifunctional proteins originally identified as negative adaptors of G protein-coupled receptors (GPCRs). Emerging evidence has also indicated that $\beta$-arrestins can activate signaling pathways independent of GPCR activation. This study was to elucidate the role of $\beta$-arrestins in diabetic nephropathy (DN) and hypothesized that $\beta$-arrestins contribute to diabetic renal injury by mediating podocyte autophagic process. We first found that both $\beta$-arrestin- 1 and $\beta$-arrestin-2 were upregulated in the kidney from streptozotocin-induced diabetic mice, diabetic $d b / d b$ mice and kidney biopsies from diabetic patients. We further revealed that either $\beta$-arrestin-1 or $\beta$-arrestin-2 deficiency (Arrb1 $1^{-/-}$or Arrb2 ${ }^{--}$) ameliorated renal injury in diabetic mice. In vitro, we observed that podocytes increased both $\beta$-arrestin- 1 and $\beta$-arrestin-2 expression levels under hyperglycemia condition and further demonstrated that $\beta$-arrestin-1 and $\beta$-arrestin-2 shared common mechanisms to suppress podocyte autophagy by negative regulation of ATG12-ATG5 conjugation. Collectively, this study for the first time demonstrates that $\beta$-arrestin- 1 and $\beta$-arrestin-2 mediate podocyte autophagic activity, indicating that $\beta$-arrestins are critical components of signal transduction pathways that link renal injury to reduce autophagy in DN. Modulation of these pathways may be an innovative therapeutic strategy for treating patients with DN.

Cell Death and Disease (2016) 7, e2183; doi:10.1038/cddis.2016.89; published online 7 April 2016

Although multiple approach targeting blood pressure, inflammation and the levels of glucose and insulin has been used in clinical trials, there is no effective therapy available to fully prevent the onset and progression of diabetic nephropathy (DN). Therefore, it is urgent to identify new therapeutic targets and develop an effective strategy for the treatment of DN. Autophagy is a catabolic process that degrades damaged proteins and organelles in mammalian cells and has a vital role in maintaining cellular homeostasis. To date, 36 autophagyrelated genes (ATGs) are identified, which have revealed dynamic and diverse mechanisms to orchestrate autophagy induction, autophagosomal membrane nucleation, elongation, closure and maturation. ${ }^{1,2}$ Emerging evidence has indicated that autophagy regulates many critical aspects of normal and disease conditions in the kidney ${ }^{3}$ and is especially important for the maintenance of postmitotic cells, such as podocytes. ${ }^{4}$ Podocyte-specific deletion of ATG5 leads to proteinuria and glomerulopathy in aging mice. ${ }^{3,5}$ Mice with podocyte-specific deletion of class III phosphoinositide 3-kinase (PI3K) vacuolar protein sorting 34 (VPS34), which is essential to initiate autophagy, exhibits substantial vacuolization, foot process effacement and progressive glomerulosclerosis. ${ }^{6}$ These findings indicate that autophagy is a key homeostatic mechanism to maintain podocyte integrity and function.
Although studies have implicated that autophagy machinery is involved in the pathogenesis of $\mathrm{DN},{ }^{7,8}$ the precise roles of autophagy and related regulatory mechanisms are largely unknown.

$\beta$-Arrestins (Arrbs) are multifunctional proteins originally identified as negative adaptors of $\mathrm{G}$ protein-coupled receptors (GPCRs) by regulation of their desensitization and internalization. Recent studies have also demonstrated that $\beta$-arrestins function to activate signaling cascades independently of GPCR activation. ${ }^{9}$ They can act as scaffold proteins that aggregate various intracellular proteins to initiate complex signaling pathways, such as mitogen-activated protein kinase, c-Jun N-terminal kinase and nuclear factor-kappa B cascades. The family of $\beta$-arrestins consists of four members, arrestins 1 and 4 that are exclusively confined to the cones and rodes of the retina and arrestins 2 and 3 ( $\beta$-arrestin- 1 (Arrb1) and $\beta$-arrestin-2 (Arrb2), respectively, that are universally expressed in all mammalian tissues. ${ }^{10}$ In the kidney, $\beta$-arrestin-2 can mediate nephrin endocytosis and impair slit diaphragm integrity. ${ }^{11,12}$ In this study, we identify for the first time that $\beta$-arrestins mediate podocyte autophagy in DN that provides a novel molecular mechanism of slit diaphragm distortion. A better understanding of the function of $\beta$-arrestins

\footnotetext{
1Department of Pharmacology, Shandong University School of Medicine, Jinan 250012, China; '2Department of Nephrology, Union Hospital, Tongji Medical College, Huazhong University of Science and Technology, Wuhan 430022, China; ${ }^{3}$ Department of Physiology, Shandong University School of Medicine, Jinan 250012, China; ${ }^{4}$ Department of Pharmacology and Toxicology, Medical College of Virginia, Virginia Commonwealth University, Richmond, VA 23298, USA; ${ }^{5}$ Department of Biochemistry and Molecular Biology, Shandong University School of Medicine, Jinan 250012, China and ${ }^{6}$ Institute of Nephrology, Shandong University, Jinan 250012, China ${ }^{*}$ Corresponding author: J Sun, Department of Biochemistry and Molecular Biology, Shandong University School of Medicine, 44\#, Wenhua Xi Road, Jinan 250012, China. Tel: 860531 88382616; Fax: 860531 88382616; E-mail: sunjinpeng@sdu.edu.cn

or F Yi, Department of Pharmacology, Shandong University School of Medicine, 44\#, Wenhua Xi Road, Jinan 250012, China. Tel: +86 053188382616 ; Fax: +86 0531 88382616; E-mail: fanyi@sdu.edu.cn

Abbreviations: GPCR, G protein-coupled receptor; PI3K, phosphoinositide 3-kinase; VPS34, vacuolar protein sorting 34; siRNA, small interfering RNA; STZ, streptozotocin; WT, wild type; DN, diabetic nephropathy; HG, high glucose; AGE, advanced glycation end-product; TEM, transmission electron microscopy Received 13.11.15; revised 01.3.16; accepted 03.3.16; Edited by E Baehrecke
} 


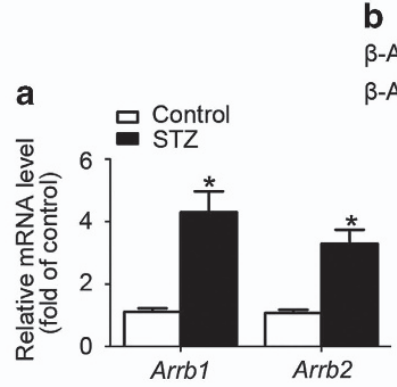

b

B-Arrestin-1 B-Arrestin-2 GAPDH

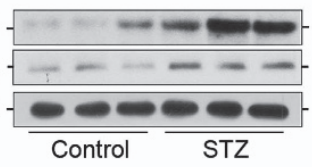

$50 \mathrm{KDa}$ $47 \mathrm{KDa}$ $37 \mathrm{KDa}$

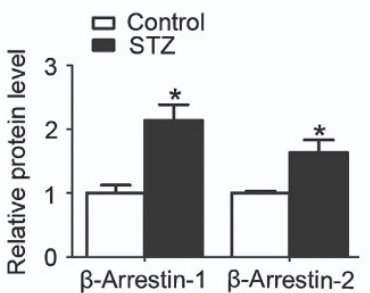

d

B-Arrestin-1 $-(-\infty \mathrm{KDa}$ $\beta$-Arrestin-2 $=-47 \mathrm{KDa}$ GAPDH $\overline{d b /+ \text { mice }} \overline{d b / d b \text { mice }}$ $37 \mathrm{KDa}$

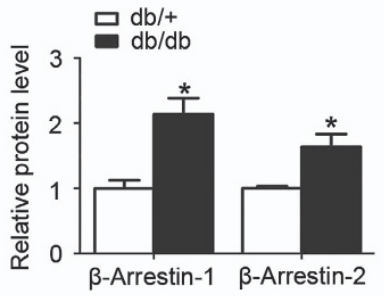

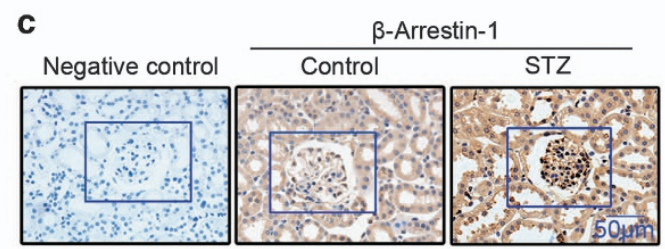
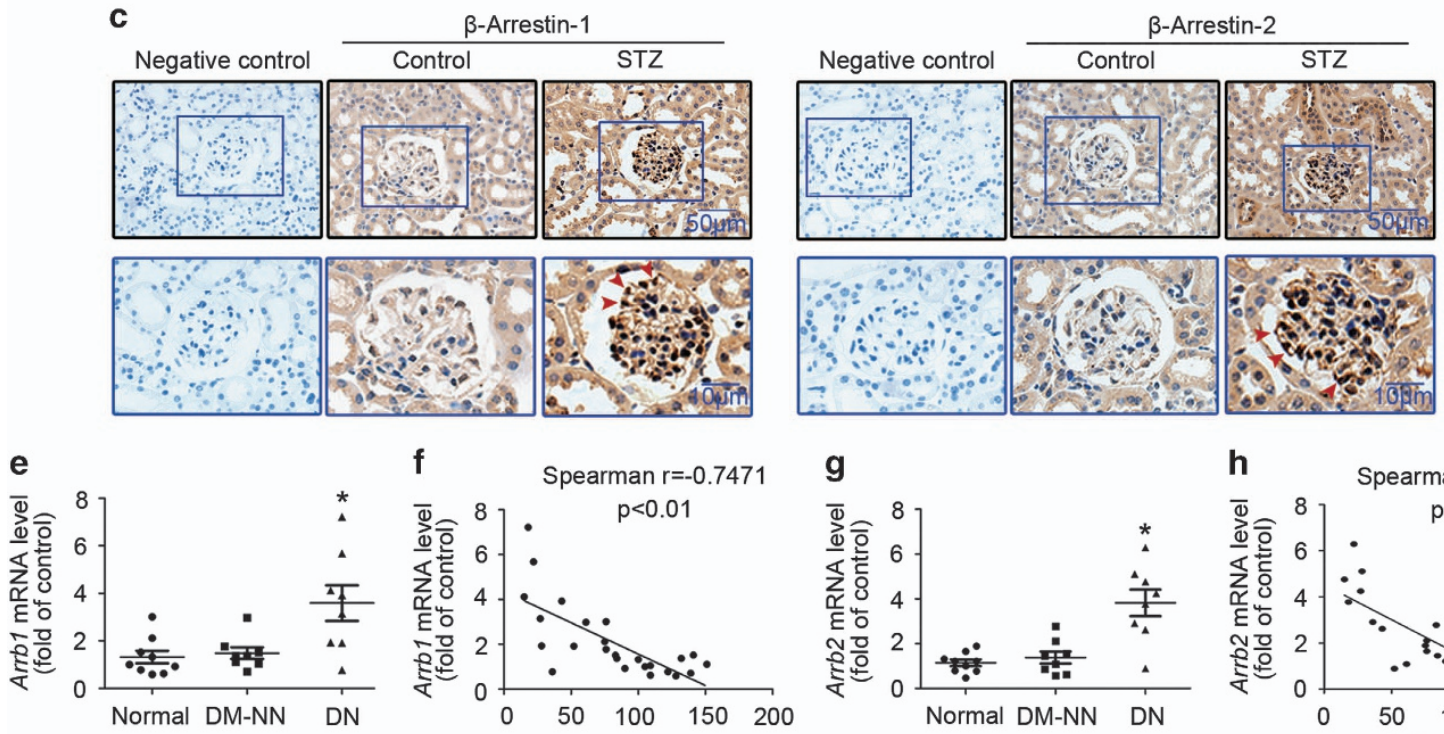

\section{$\mathbf{f}$}
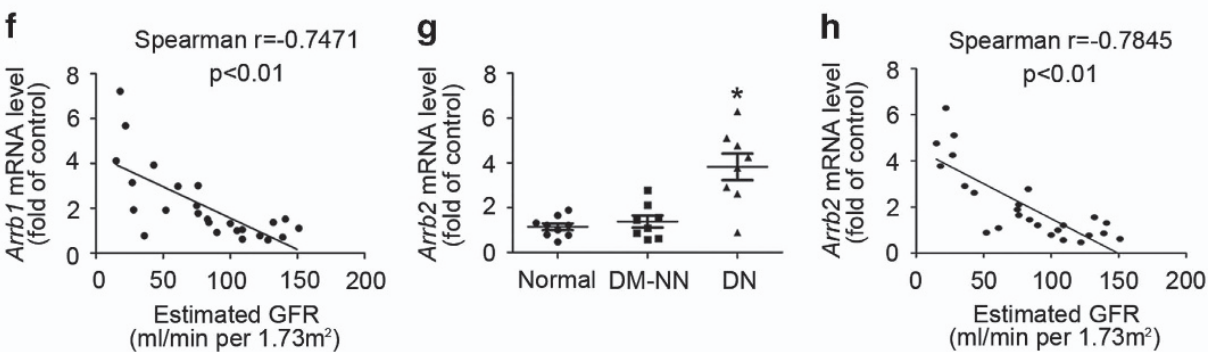

Figure 1 Upregulation of $\beta$-arrestin-1 and $\beta$-arrestin-2 in the kidney from STZ-induced diabetic mice, diabetic $d b / d b$ mice and kidney biopsies from diabetic patients. (a) Relative mRNA levels of Arrb1 and Arrb2 in the kidney from STZ-induced diabetic mice (mean \pm S.E.M.). (b) Representative western blotting gel documents and summarized data showing the relative protein levels of $\beta$-arrestin-1 and $\beta$-arrestin-2 in the kidney from STZ-induced diabetic mice (mean \pm S.E.M.). (c) Representative images are from ISH of $\beta$-arrestin-1 and $\beta$-arrestin-2 in the kidney from STZ-induced diabetic mice (arrowheads: representative podocytes). Scramble probes were used as negative controls. (d) Representative western blotting gel documents and summarized data showing the relative protein levels of $\beta$-arrestin-1/2 in the kidney from diabetic $d b / d b$ mice and their genetic control $d b /+$ mice (mean \pm S.E.M.). (e) Relative mRNA levels of Arrb1 in from normal subjects $(n=9)$ and patients with DN $(n=8)$ or DM-NN $(n=8)$ (mean \pm S.E.M.). (f) Negative correlation (Spearman $r=-0.7471, P<0.05$ ) between Arrb1 mRNA levels and estimated glomerular filtration rate (eGFR) in all subjects. (g) Relative mRNA levels of Arrb2 in the renal biopsies from different patients (mean \pm S.E.M.). (h) Negative correlation (Spearman $r=-0.7845, P<0.05$ ) between Arrb2 mRNA levels and eGFR in all subjects. ${ }^{*} P<0.05$ versus normal subjects

in the kidney will provide unexpected opportunities for developing new therapies for DN.

\section{Results}

Upregulation of $\beta$-arrestin-1 and $\beta$-arrestin-2 in the kidney from STZ-induced diabetic mice, diabetic $d b / d b$ mice and kidney biopsies from diabetic patients. As shown in Supplementary Table S1, STZ-induced diabetic mice had hyperglycemia and lower body weight compared with their non-diabetic counterparts, and no difference in blood pressure was observed among these groups. Real-time RTPCR (Figure 1a) and western blotting (Figure 1b) analyses showed that both $\beta$-arrestin-1 and $\beta$-arrestin-2 were upregulated in the kidney from diabetic mice, which was further confirmed in paraffin-embedded sections of kidney tissues by in situ hybridization (ISH; Figure 1c) or immunohistochemical staining (Supplementary Figure S1a). We also observed the upregulation of $\beta$-arrestin- 1 and $\beta$-arrestin- 2 in the kidney in another in vivo model of diabetes, the $d b / d b$ mice, indicating that $\beta$-arrestin-1 and $\beta$-arrestin- 2 may be the common pathogenic factors in DN (Figure 1d). In consistent with the changes in animal studies, real-time RT-PCR (Figures 1e-g) and immunohistochemical staining (Supplementary Figure $\mathrm{S} 1 \mathrm{~b})$ further confirmed the upregulation of $\beta$-arrestin1 and $\beta$-arrestin-2 in the human diabetic renal tissues compared with normal controls and diabetic patients without nephropathy (DM-NN). We further found that both Arrb1 (Spearman $r=-0.7471, P<0.01$; Figure 1f) and Arrb2 (Spearman $r=-0.7845, P<0.01$; Figure 1h) mRNA levels 
a

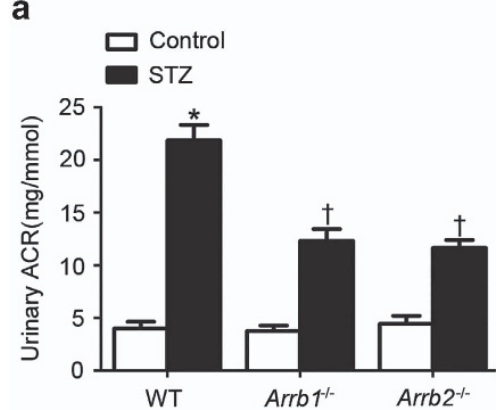

b

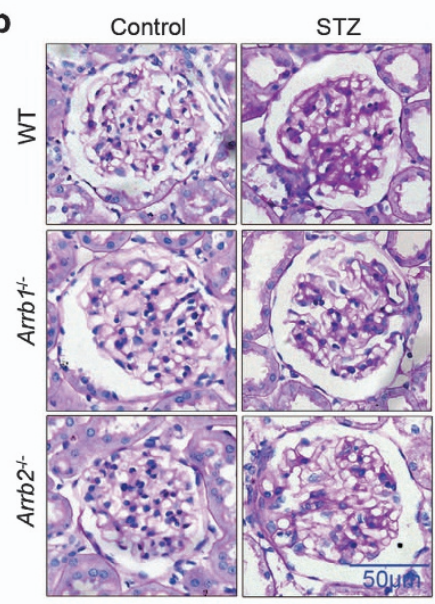

C

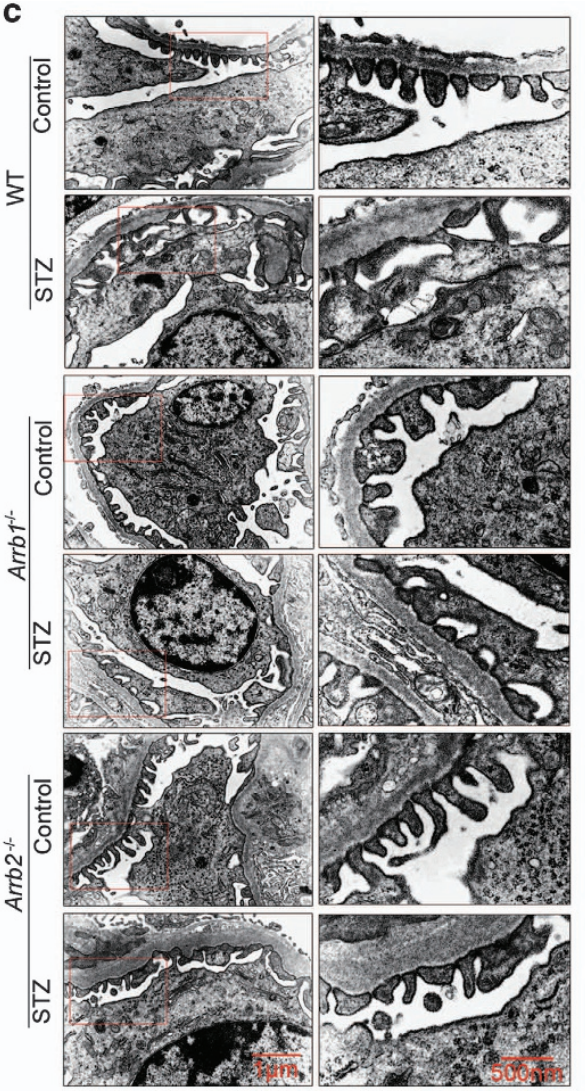

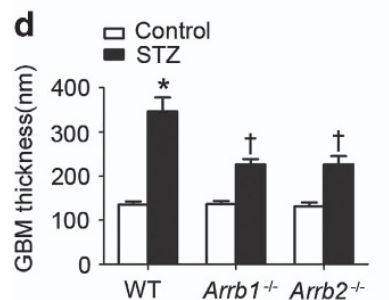
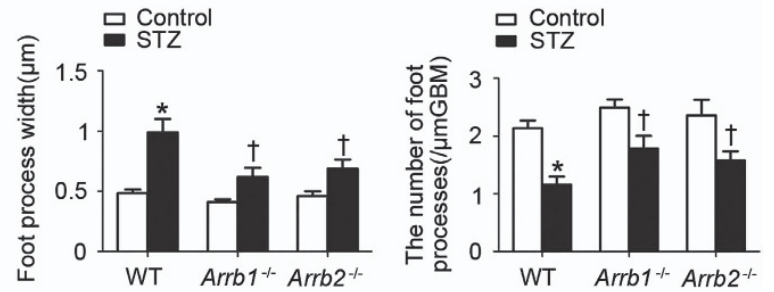

Figure 2 Either Arrb1 or Arrb2 deficiency ameliorated renal injury in diabetic mice. (a) Urinary ACR in different groups of mice. (b) Representative photomicrographs showing typical glomerular structure changes in different groups of mice. (c) Representative TEM images showing morphological changes in the podocyte foot process in different groups of mice. (d) Indices for glomerular filtration barrier integrity, including GBM thickness, foot process width and the number of foot processes $/ \mu \mathrm{m} \mathrm{GBM} \mathrm{(mean} \pm$ S.E.M.; $n=8$; ${ }^{*} P<0.05$ versus control, $\dagger P<0.05$ versus WT diabetic mice

were negatively correlated with estimated glomerular filtration rate in all the available subjects individually.

Either Arrb1 or Arrb2 deficiency ameliorated renal injury in diabetic mice. As shown in Figure $2 a$, urinary albumin-tocreatinine ratio (ACR) was significantly reduced in uninephrectomized (Unx) Arrb1 or Arrb2-deficient $\left(A r r b 1^{-1-}\right.$ or $\mathrm{Arb2}^{-1}$ ) diabetic mice. In wild-type (WT) diabetic mice, morphological examinations showed that the glomerular mesangium was expanded with hypercellularity and capillary collapse in the glomerulus (Figure $2 b$ ). Transmission electron microscopy (TEM) analyses further revealed podocyte injuries in diabetic mice (Figures $2 c$ and d). The glomerular basement membrane (GBM) under the foot process effacement was thickened and the three-layered structure was lost. The number of foot processes along the GBM that was significantly decreased paralleled the increase in podocyte foot process width in diabetic mice. All of which could be ameliorated by Arrb1 or Arrb2 deficiency.

$\beta$-Arrestin-1 and $\beta$-arrestin-2 were upregulated in podocytes under hyperglycemia condition both in vivo and in vitro. First, immunofluorescent results further confirmed that both $\beta$-arrestin- 1 and $\beta$-arrestin- 2 expression levels were increased in podocytes from diabetic mice (Figure 3a). We then detected the expression patterns in podocytes treated with high glucose (HG, Figure $3 b)$, advanced glycation endproducts (AGEs, Figure $3 c$ ) or transforming growth factor- $\beta 1$ (TGF- $\beta 1$, Figure $3 d$ ) (the common detrimental factors in DN) in vitro. Our results revealed that all these stimuli significantly increased the expression levels of podocyte $\beta$-arrestin- 1 and $\beta$-arrestin-2 in a concentration-dependent manner. 
a

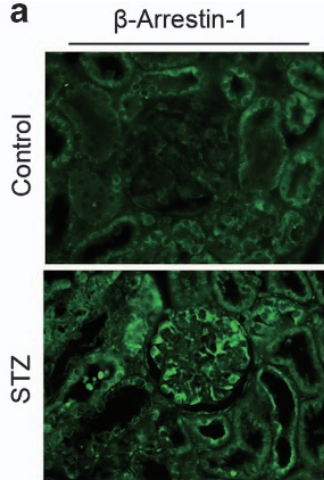

$\beta$-Arrestin-2
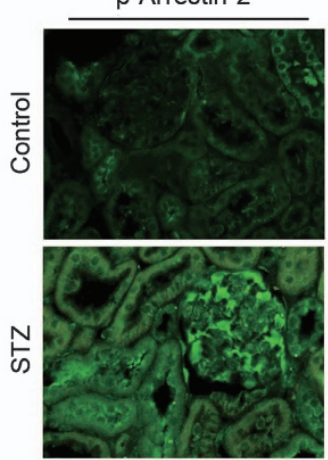

b
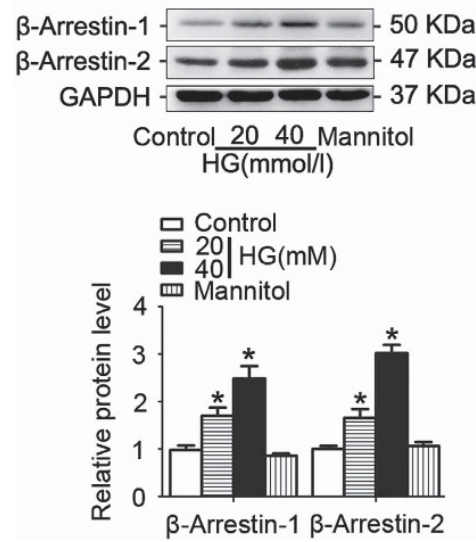

Synaptopodin

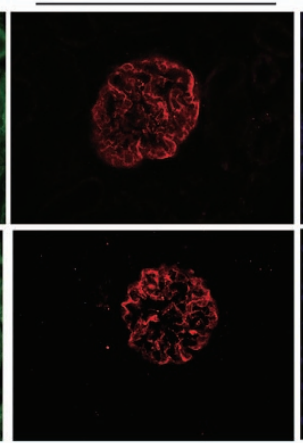

Synaptopodin
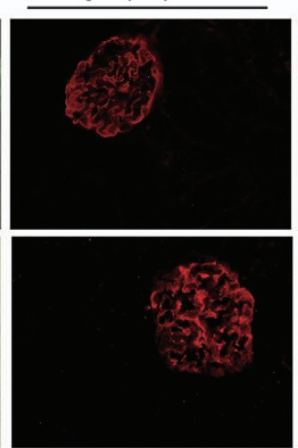

C

$\beta$-Arrestin-1 $-\longrightarrow-50 \mathrm{KDa}$

B-Arrestin-2 $-47 \mathrm{KDa}$

GAPDH
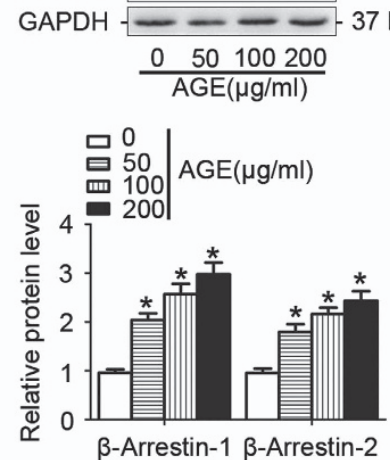

DAPI

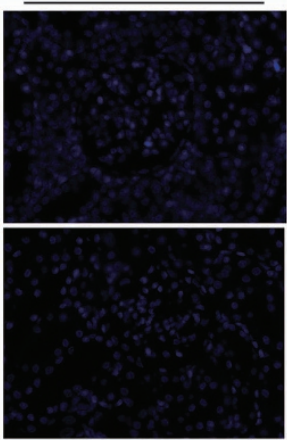

DAPI
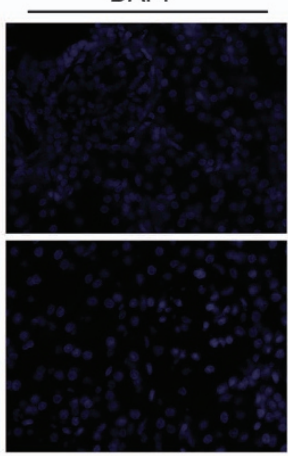

d
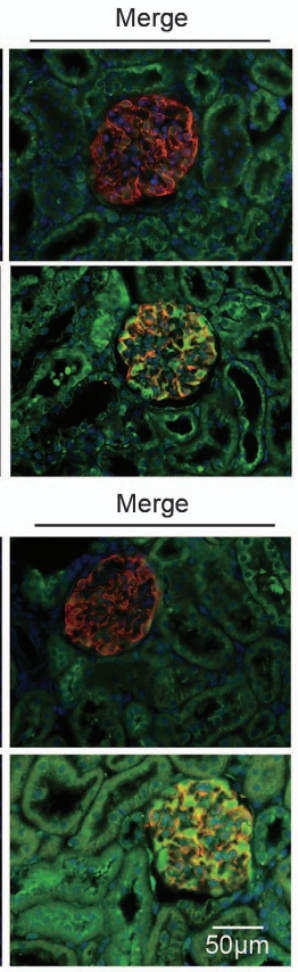
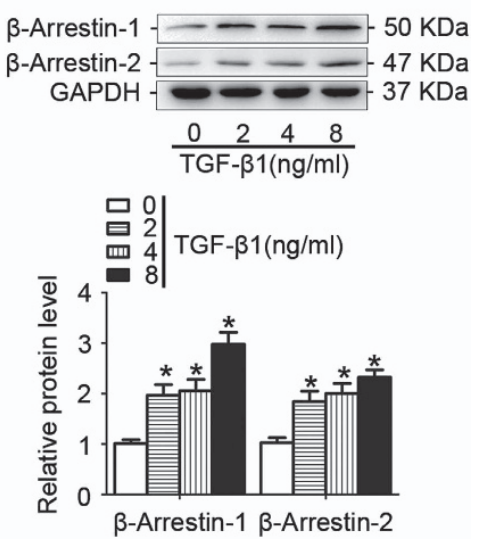

Figure $3 \beta$-Arrestin-1 and $\beta$-arrestin-2 were upregulated in podocytes under hyperglycemia both in vivo and in vitro. (a) Representative confocal microscopic images showing the upregulation of podocyte $\beta$-arrestin-1/2 in the kidney from STZ-induced diabetic mice; synaptopodin was used as a podocyte marker. (b) Representative western blotting gel documents and summarized data showing the protein levels of $\beta$-arrestin-1/2 in podocytes treated with $\mathrm{HG}$ (final concentration 20 or $40 \mathrm{mmol} / \mathrm{l}$ in medium) for $24 \mathrm{~h}$. (c) Representative western blotting gel documents and summarized data showing the protein levels of $\beta$-arrestin-1/2 in podocytes with AGE (50-200 $\mu \mathrm{g} / \mathrm{ml})$ for $24 \mathrm{~h}$. (d) Representative western blotting gel documents and summarized data showing the protein levels of $\beta$-arrestin-1/2 in podocytes treated with TGF- $\beta 1$ (2-8 ng/ml) for 24 h. (means \pm S.E.M.; $n=6 ;{ }^{*} P<0.05$ versus control)

\begin{abstract}
$\beta$-Arrestin-1 and $\beta$-arrestin-2 reduced basal autophagy in podocytes with HG treatment. To investigate the role of $\beta$-arrestins on the regulation of autophage in podocytes, gene silencing of Arrb1 (Figure 4a) or Arrb2 (Figure 4b) were used in this study. TEM showed that the number of typical autophagosomes with double membranes was significantly increased by gene silencing of Arrb1 or (and) Arrb2 in podocytes with $\mathrm{HG}$ treatment (Figures $4 \mathrm{c}$ and d). Moreover, autophagic flux is often inferred on the basis of LC3-II turnover, measured by western blotting in the presence of lysosomal inhibitors such as chloroquine and bafilomycin $\mathrm{A} 1$
\end{abstract}

that elevate/neutralize the lysosomal/vacuolar $\mathrm{pH}$. We found that gene silencing of Arrb1 increased the LC3-II/LC3-I conversion and the amount of LC3-II was much higher in the presence of chloroquine and bafilomycin $\mathrm{A} 1$, indicating that autophagic flux was occurring (Figure 4e). Finally, we utilized the tandem RFP-GFP-LC3 adenovirus construct to monitor autophagic flux as described. ${ }^{7,13}$ This assay is based on the $\mathrm{pH}$ difference between the acidic autolysosome and the neutral autophagosome. In addition, green fluorescent protein (GFP) signal is sensitive to the acidic and/or proteolytic conditions of the lysosome lumen, whereas red 


\section{a}

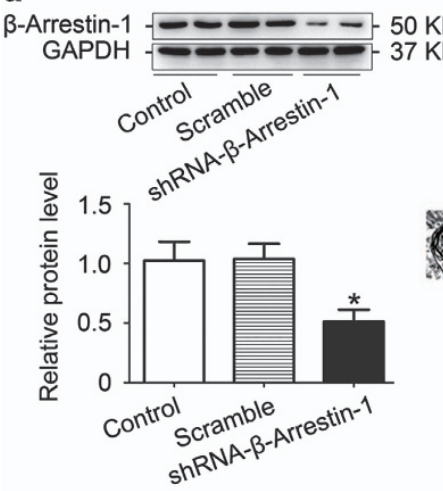

b

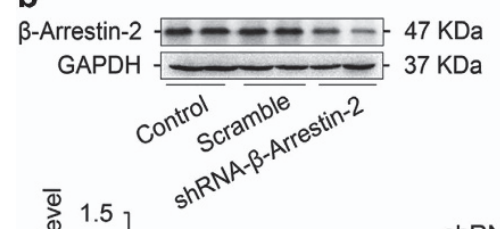

c

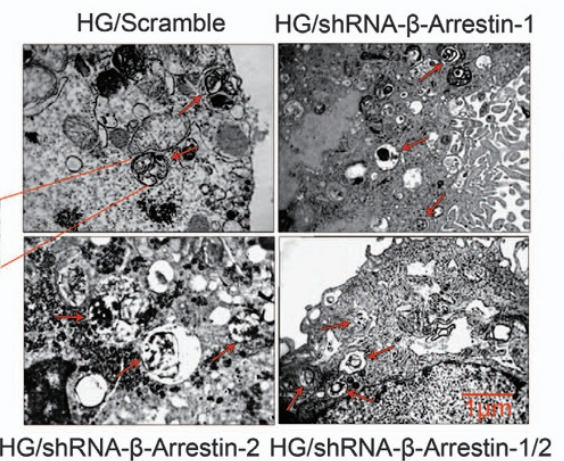

d

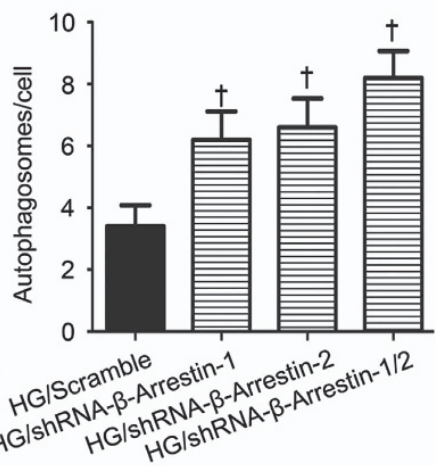

e

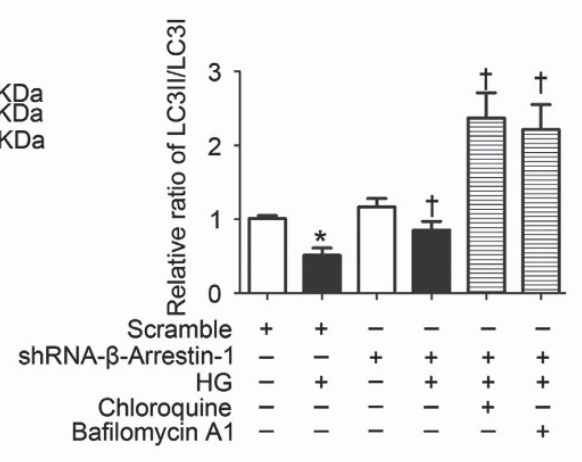

\section{LC3.} GAPDH

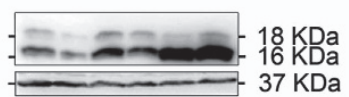

$\mathrm{HG}-+-++$

Chloroquine - - - +

Bafilomycin A1 - - - - +

f

Scramble $++-\overline{-}-$
shRNA- $\beta$-Arrestin-1 -+
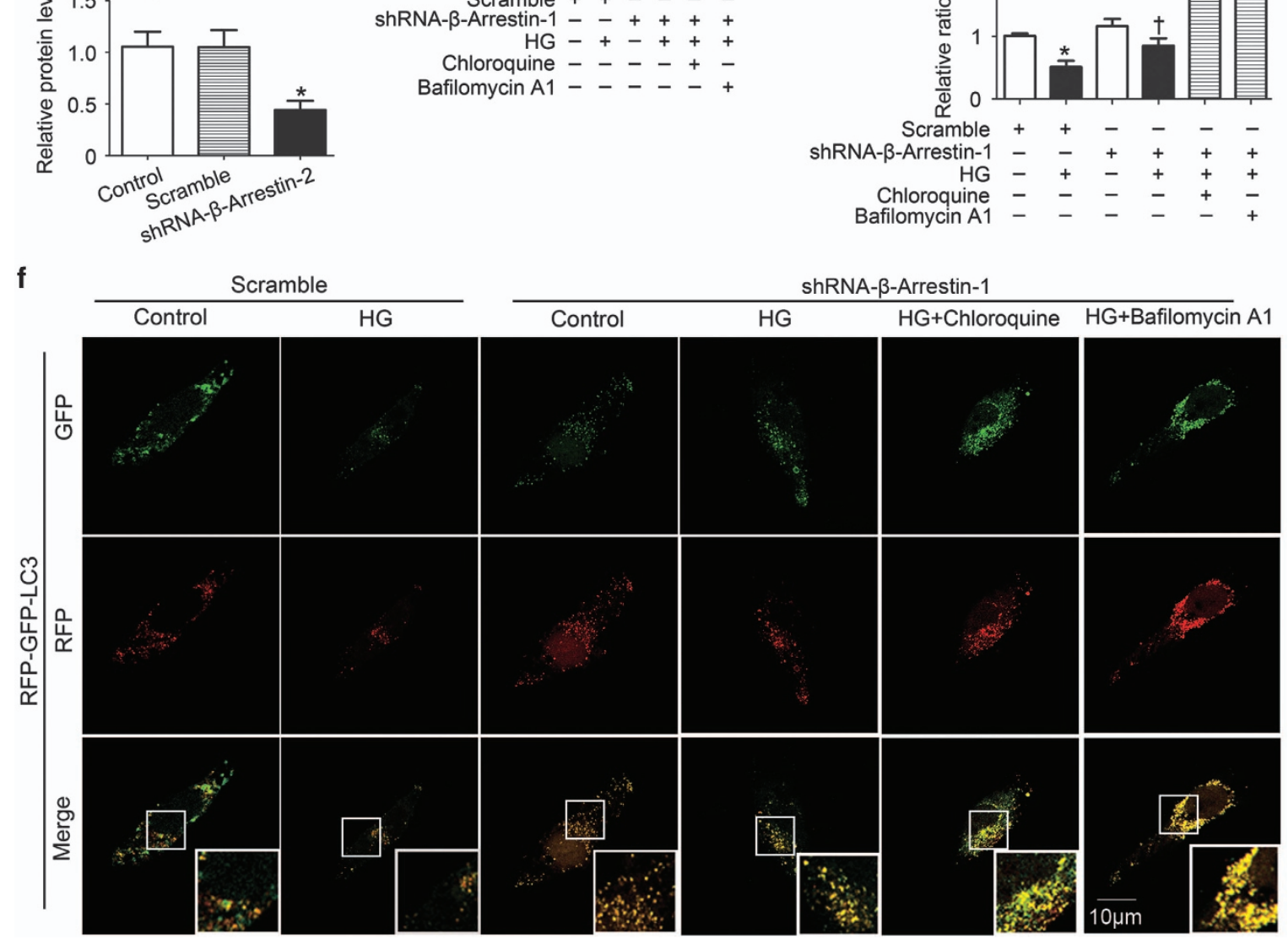

Figure $4 \quad \beta$-Arrestin-1 and $\beta$-arrestin-2 reduced basal autophagy in podocytes with HG treatment. (a) Representative western blotting gel documents and summarized data showing the efficiency of Arrb1 knockdown by shRNA- $\beta$-arrestin-1 transfection. (b) Representative western blotting gel documents and summarized data showing the efficiency of Arrb2 knockdown by shRNA- $\beta$-arrestin-2 transfection. (c) Representative electronic micrographs showing autophagosomes in HG-treated podocytes with or without gene silencing of Arrb1 or (and) Arrb2. The arrows indicate autophagosomes. (d) The number of autophagic vesicles (AV) was determined with 10 cells in each sample, respectively. (e) Representative western blotting gel documents and summarized data show the levels of LC3-II/LC3-I in the presence of lysosomal inhibitors such as chloroquine (30 $\mu$ mol/I) and bafilomycin A1 (50 nmol/l) after Arrb1 knockdown. (f) Representative images of LC3 staining by measurement of fluorescent intensity in podocytes in different groups of podocytes infected with RFP-GFP-LC3 adenovirus for $24 \mathrm{~h}$ (means \pm S.E.M.; $n=6 ;{ }^{*} P<0.05$ versus control, $\uparrow P<0.05$ versus scramble of HG treatment) 

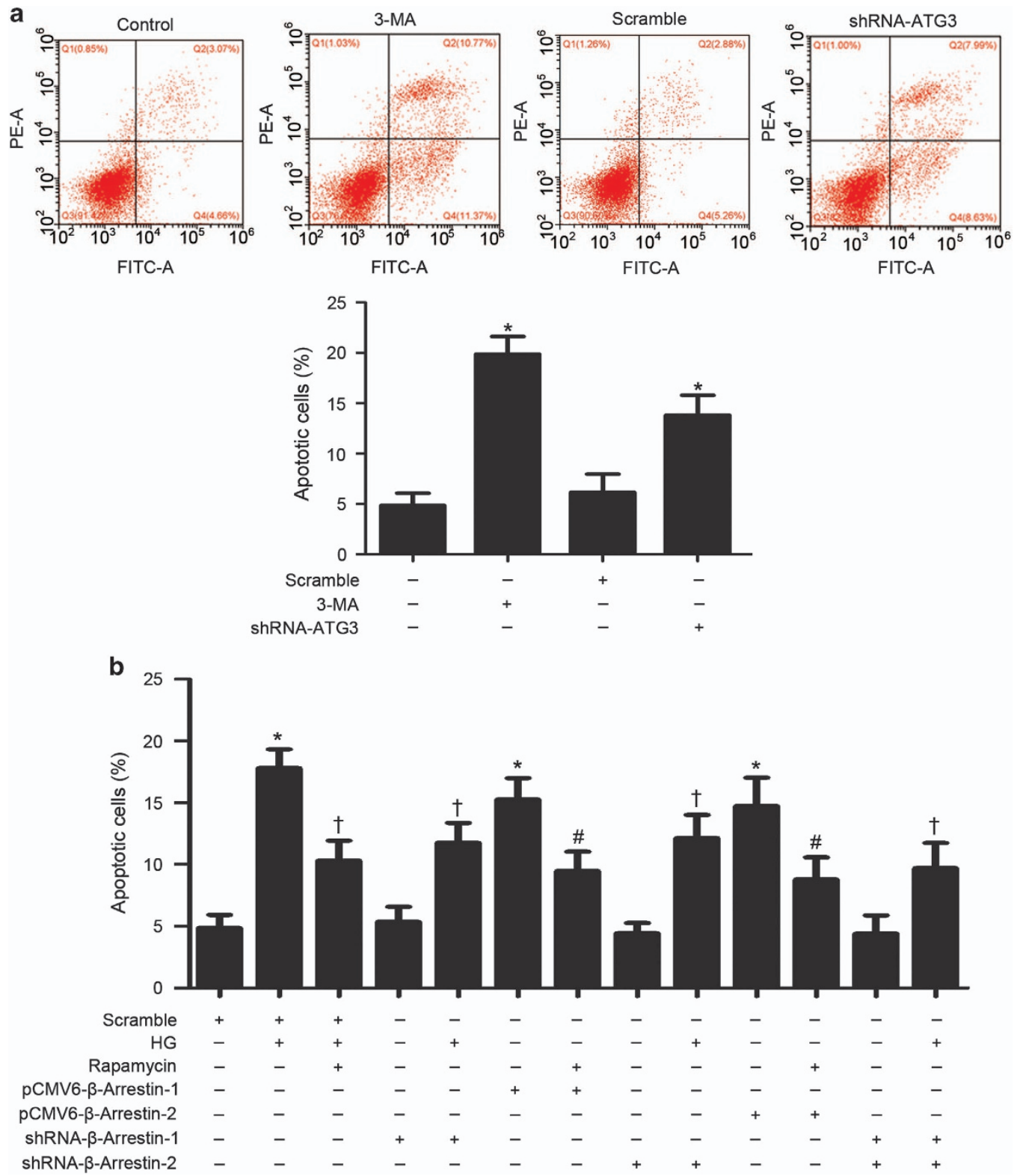

Figure 5 Autophagy inhibition by $\beta$-arrestins reduced podocyte viability by flow cytometric analysis. (a) Autophagy inhibition by gene silencing of ATG-3- or 3-methyladenine (3-MA)-induced apoptosis. (b) HG-induced apoptosis was alleviated by gene silencing of $\beta$-arrestins as well as by restoring defective autophagy with low dose of rapamycin. Overexpression of $\beta$-arrestins by pCMV6- $\beta$-arrestin-1 or pCMV6- $\beta$-arrestin-2 transfection in podocytes induced apoptosis, which could be attenuated by rapamycin. (means \pm S.E.M.; $n=6$; ${ }^{*} P<0.05$ versus control, $\dagger P<0.05$ versus scramble of $\mathrm{HG}$ treatment, $\# P<0.05$ versus overexpression of $\beta$-arrestins treatment)

fluorescent protein (RFP) is relatively stable. Therefore, colocalization of both GFP and RFP fluorescence indicates a compartment that has not fused with a lysosome, such as the phagophore or an autophagosome. When an autophagosome fuses with a lysosome to form autolysosomes, the GFP moiety degrades. In Figure 4f, we observed the successful introduction of the RFP-GFP-LC3 adenovirus construct showing both fluorescent proteins. In addition to accumulation of LC3, more red puncta were present in podocytes transfected with shRNA- $\beta$-arrestin- 1 under HG conditions. In the presence of chloroquine and bafilomycin A1, GFP-LC3positive structures and yellow signals that results from merging the red and green channels were further increased.
Similar results were also found from podocytes transfected with shRNA- $\beta$-arrestin-2 (data not shown).

Autophagy inhibition by $\boldsymbol{\beta}$-arrestins reduced podocyte viability. To examine the role of autophagy in podocytes, autophagy inhibition by gene silencing of ATG-3 or 3-methyladenine (3-MA), an autophagy inhibitor, was used in this study. We found that autophagy inhibition induced apoptosis by flow cytometric analysis, indicating that autophagy has beneficial effects on the viability of podocytes (Figure 5a). Furthermore, we examined whether $\beta$-arrestins regulate podocyte viability via autophagy, and an autophagy enhancer rapamycin was used as a control to restore 
a

IP: $\beta$-Arrestin-1

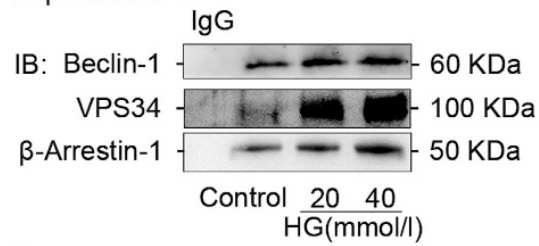

C

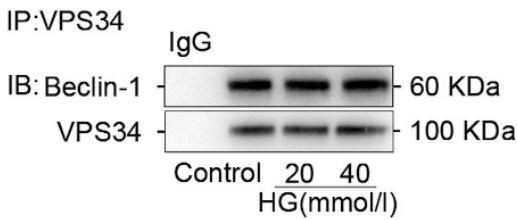

d

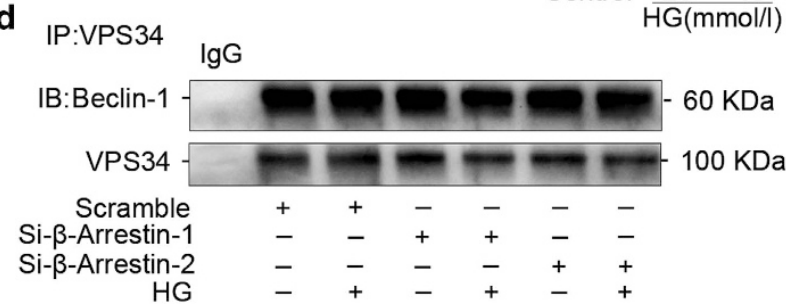

b

IP: $\beta$-Arrestin-2

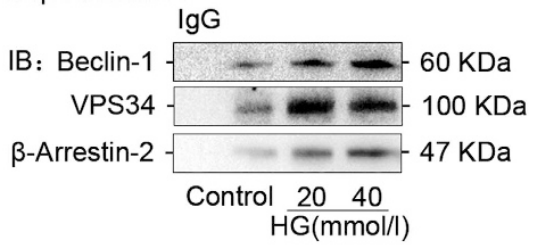

Input:

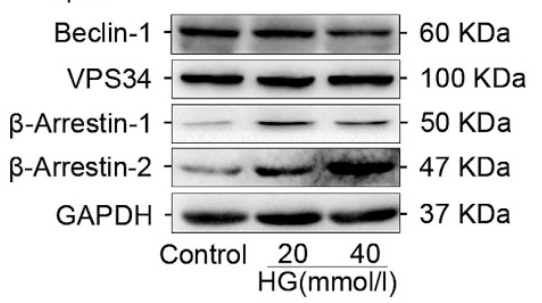

Figure $6 \quad \beta$-Arrestin-1 and $\beta$-arrestin-2 had not participated in the formation of PI3K core complex. (a) Immunoprecipitation assays showing that $\beta$-arrestin-1 interacted with VPS34 and beclin-1 and the interaction between VPS34 and $\beta$-arrestin-1 was significantly enhanced in podocytes with HG treatment. (b) An increased tendency was also observed for the interaction between VPS34 and $\beta$-arrestin-2. (c) There was no interaction change between beclin-1 and VPS34 in podocytes with HG treatment. (d) Gene silencing of Arrb1 or Arrb2 had no effects on the formation of PI3K core complex in podocytes

autophagy. Our study showed that HG-induced apoptosis was alleviated by gene silencing of $\beta$-arrestins as well as by restoring defective autophagy with low dose of rapamycin. Consistently, overexpression of $\beta$-arrestins induced apoptosis, which could be attenuated by rapamycin. Collectively, our results suggested that basal autophagy is essential in maintaining podocyte viability and that the inhibition of autophagy by $\beta$-arrestins may have a severe negative impact on podocyte function.

$\beta$-Arrestin-1 and $\beta$-arrestin-2 were interacted with beclin-1 and VPS34 individually, but had no effects on the formation of PI3K core complex in podocytes under HG condition. To explore the mechanisms by which $\beta$-arrestins mediated autophagy, we first investigated whether the class III PI3K core complex, which is required for autophagosome formation, can be regulated by $\beta$-arrestins. In this complex, VPS34 is the class III PI3K that phosphorylates phosphatidylinositol to generate phosphatidylinositol 3-phosphate, which is essential for both intracellular trafficking and autophagosome formation, and beclin 1 is part of a class III PI3K complex and participates in autophagosome formation by regulation of VPS34 activity. ${ }^{14}$ Immunoprecipitation assay showed that $\beta$-arrestin- 1 interacted with VPS34 and beclin-1 and the interaction between VPS34 and $\beta$-arrestin- 1 was significantly enhanced in podocytes with $\mathrm{HG}$ treatment (Figure 6a). Similarly, an increased tendency was also observed for the interaction between VPS34 and $\beta$-arrestin-2
(Figure 6b). However, there was no interaction change between belcin-1 and VPS34 in podocytes with HG treatment (Figure 6c) as well as gene silencing of Arrb1 or Arrb2 (Figure $6 \mathrm{~d}$ ). These results indicated that neither $\beta$-arrestin-1 nor $\beta$-arrestin-2 had effects on the formation of PI3K core complex under HG condition in podocytes.

$\beta$-Arrestin-1 and $\beta$-arrestin-2 negatively regulated ATG12-ATG5 conjugation system in podocytes with HG treatment. The LC3-II/LC3-I ratio usually correlates with the bulk autophagic flux and autophagosome formation, and the levels of the ATG12-ATG5 conjugate is used as a measure of effectiveness in early stages of autophagy leading up to autophagosome formation. ${ }^{15}$ Therefore, we further detected the effect of $\beta$-arrestins on ATG12-ATG5 conjugation and LC3 processing. It was found that $\mathrm{HG}$ dramatically reduced ATG12-ATG5 conjugation and LC3-II/LC3-I ratio, which can be restored by gene silencing of Arrb1 (Figure 7a) or Arrb2 (Figure 7b). Consistently, overexpression of Arrb1 (Supplementary Figure S2a) or Arrb2 (Supplementary Figure S2b) significantly reduced ATG12-ATG5 levels and LC3-II/LC3-I ratio. These results further confirmed the role of $\beta$-arrestins in autophagosome formation. Notably, ATG7, an E1-like enzyme that is essential for ATG12-ATG5 conjugation and LC3-II/LC3-I conversion, interacted with $\beta$-arrestin-1 and $\beta$-arrestin-2 (Figure 7c), which could be strengthened by HG treatment. Consistently, we found that the LC3-II/LC3-I ratio and ATG12-ATG5 levels were recovered in the kidney of 

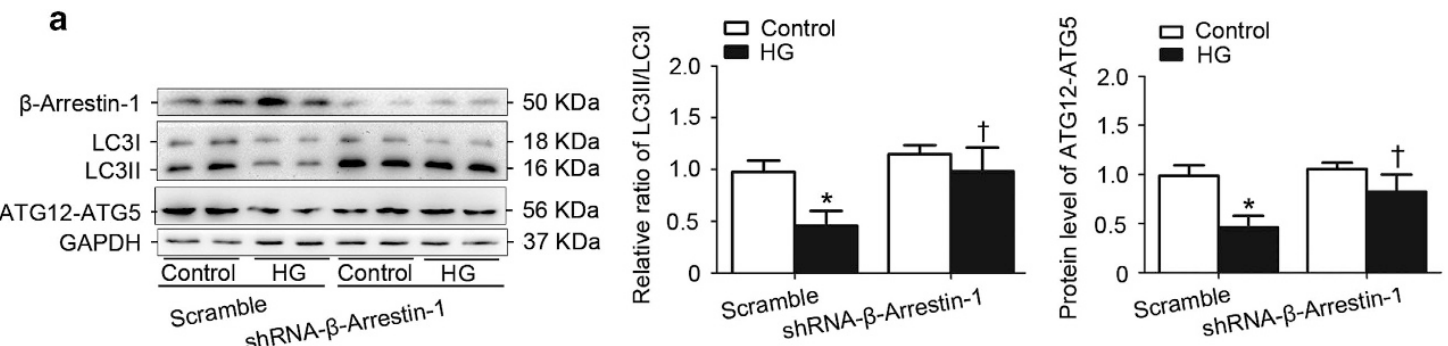

b
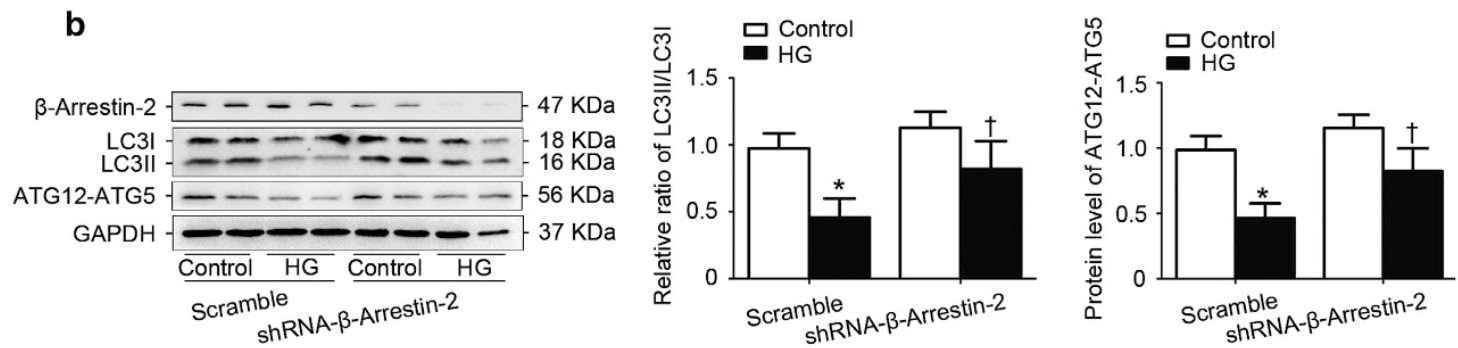

C

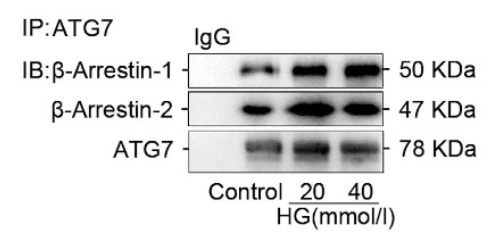

d

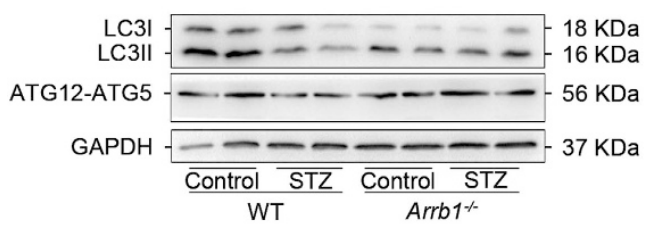

e

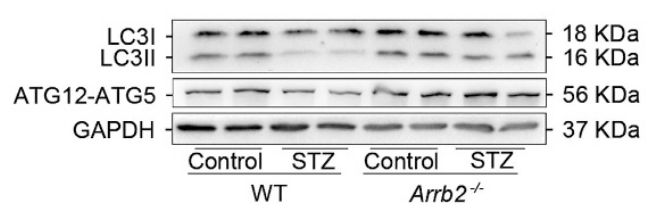

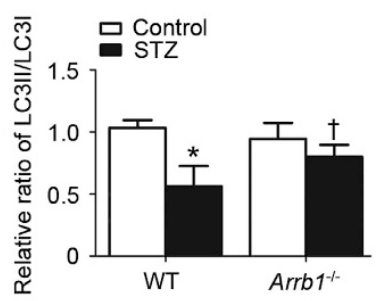
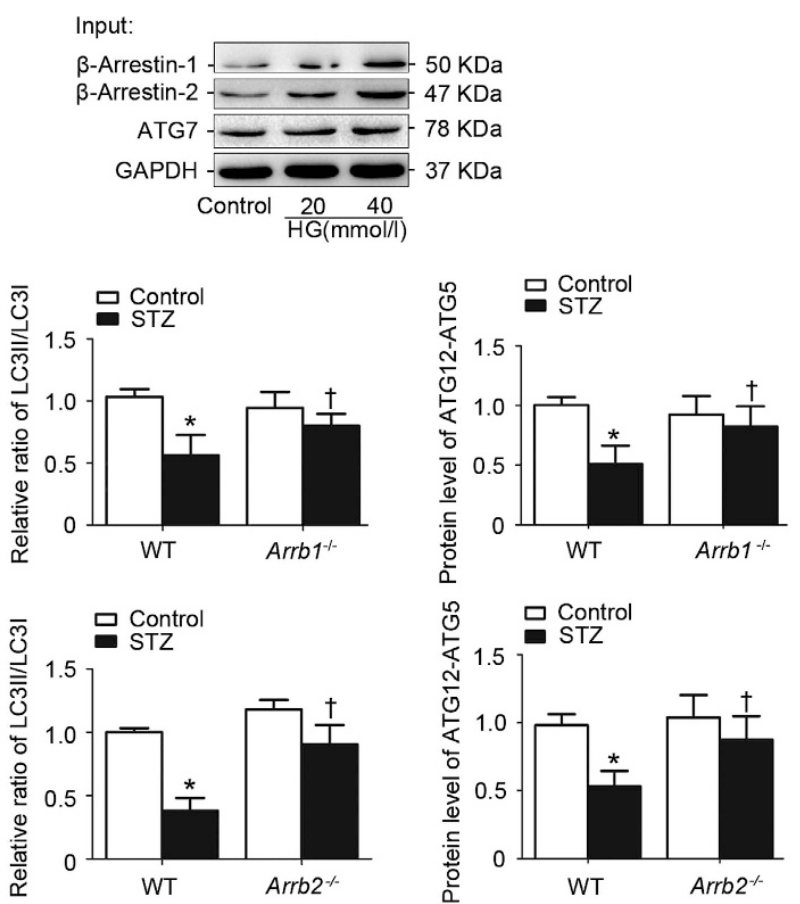

Figure $7 \quad \beta$-Arrestin-1 and $\beta$-arrestin-2 negatively regulated ATG12-ATG5 conjugation system in podocytes with HG treatment. (a) Representative western blotting gel documents and summarized data showing the effect of Arrb1 knockdown on the levels of ATG12-ATG5 and LC3-II/LC3-I. (b) Representative western blotting gel documents and summarized data showing the effect of Arrb2 knockdown on the levels of ATG12-ATG5 and LC3-II/LC3-I. (c) Immunoprecipitation assay showing that $\beta$-arrestin-1/2 interacted with ATG7 and the interactions between ATG7 and $\beta$-arrestin-1/2 were significantly enhanced in podocytes with $\mathrm{HG}$ treatment. (means \pm S.E.M.; $n=6 ;{ }^{*} P<0.05$ versus control, $\dagger P<0.05$ versus scramble of $\mathrm{HG}$ treatment). (d) Representative western blotting gel documents and summarized data showing the levels of ATG12-ATG5 and LC3-II/LC3-I in the kidney from Arrb $1^{--}$diabetic mice. (e) Representative western blotting gel documents and summarized data showing the levels of ATG12-ATG5 and LC3-II/LC3-I in the kidney from Arrb2 $^{-/}$diabetic mice. Values are means \pm S.E.M.; ${ }^{*} P<0.05$ versus control, $\uparrow P<0.05$ versus WT STZ mice $(n=8)$

Arrb1 $^{-/}$(Figure 7d) or Arrb2 ${ }^{-/}$(Figure 7e) diabetic mice compared with those of WT diabetic mice in vivo.

\section{Discussion}

In this study, we identified for the first time that $\beta$-arrestin- 1 and $\beta$-arrestin-2 are upregulated in DN and that the defective autophagy and the impaired filtration barrier function in diabetic mice are alleviated by Arrb1 or Arrb2 deficiency. Although we have not examined the protective effects in the Arrb $1^{-1-} / \mathrm{Arrb}^{-1}$ double knockout mice because of embryonic lethality of these mice, these results clearly indicate the importance of $\beta$-arrestin- 1 and $\beta$-arrestin- 2 in mediating autophagy and renal function. Considering that podocyte function as GBM turnover, maintenance of the glomerular 


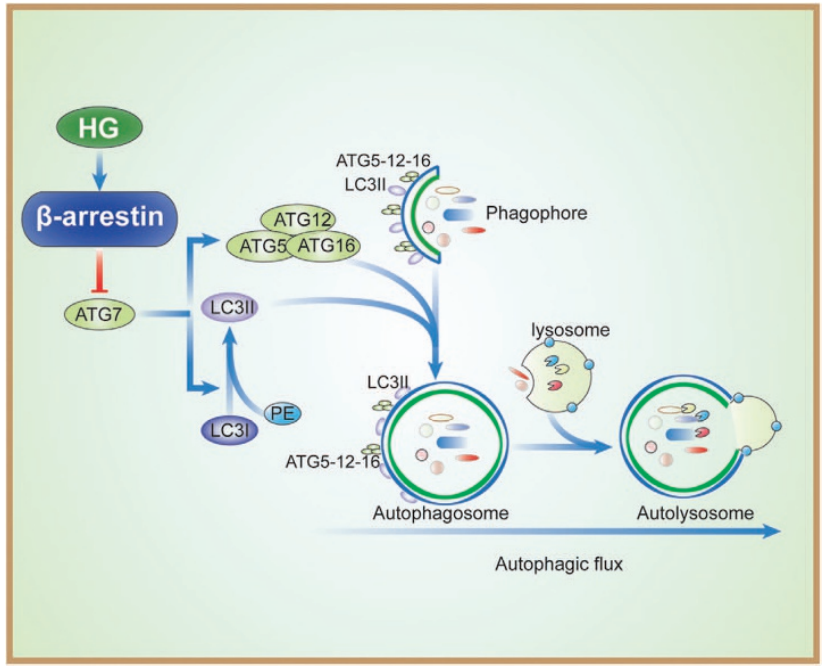

Figure 8 Proposed mechanisms by which $\beta$-arrestins negatively mediate podocyte autophagic process in DN. The enhanced interaction between $\beta$-arrestin1/2 and ATG7 may block ATG7 to freely access and activate glycine residue of ATG12, thereby reducing ATG12-ATG5 conjugation, leading to the reduction of autophagy

filtration barrier and regulation of glomerular filtration, ${ }^{16,17}$ and the high level of basal autophagy in podocytes is vital to maintain their architectural integrity, ${ }^{3}$ we therefore focus on the role of $\beta$-arrestins in diabetic-induced podocyte injury. Consistently, both $\beta$-arrestin- 1 and $\beta$-arrestin- 2 are associated with hyperglycaemia-reduced autophagy in podocytes in vitro.

The regulation of autophagy is extremely complicated and multiple signaling pathways are involved in this process. ATGs are key molecules in the core of the regulatory machinery of autophagy. ${ }^{18}$ Among them, the class III PI3K complex (beclin1-ATG14-VPS15-VPS34) has an essential role in the initiation of the autophagosome formation. Mechanically, class III PI3K, also named VPS34, is associated with and regulated by beclin-1, and the interaction of beclin-1 with VPS34 promotes the catalytic activity of VPS34, which is crucial for canonical autophagosome formation. ${ }^{19}$ Several cofactors have been identified to interact with beclin-1, such as ATG14L, Bif-1, Rubicon and Bcl-2, to regulate VPS34 activity either positively or negatively. ${ }^{20} \mathrm{~A}$ recent study has shown that $\beta$-arrestin- 1 interacts with beclin-1 and VPS34 under hypoxia condition but not under normal conditions in neurons, which contributes to promote autophagosome formation by enhanced beclin-1 and VPS34 interaction, ${ }^{21}$ thereby inducing autophagy. However, the present study displays different outcomes under hyperglycemia condition. Although we found that $\beta$-arrestin- 1 as well as $\beta$-arrestin- 2 interacts with beclin- 1 and VPS34 even in the normal condition, and $\beta$-arrestin- 1 and $\beta$-arrestin- 2 are further recruited to the PI3K core complex under HG condition, there is no effect on the interaction between beclin-1 and VPS34. The mixed observation may result from the possible different contribution of $\beta$-arrestins to autophagy in different diseases or different cell types. So how $\beta$-arrestins negatively regulate autophagy in podocytes is an intriguing question of fundamental importance. In this study, we provide potential mechanisms to answer this question. The elongation and expansion steps in autophagosome formation involve two ubiquitin-like proteins, ATG12 and ATG8/LC3. The conjugation of ATG12 to ATG5 is catalyzed by ATG7 and ATG10 (E1- and E2-like enzymes, respectively) to form covalently linked ATG12-ATG5. ${ }^{22}$ Following formation of the ATG12-ATG5 conjugate, ATG16L non-covalently associates with this conjugate to produce the ATG12-ATG5-ATG16 multimeric complex, ${ }^{23}$ which is essential for LC3-PE conjugation and proper elongation of the isolation membrane. In addition, ATG7 also interacts with E2-like enzyme, ATG3, to mediate conjugation of ATG8/LC3, which is a key step during the expansion of phagophores in autophagy. ${ }^{24}$ Therefore, ATG7 takes a center stage on the regulation of these two conjugation systems. Interestingly, we find that HG-reduced ATG12-ATG5 levels, as well as LC3-II/LC3-I ratio, can be recovered by gene silencing of Arrb1 or Arrb2. Furthermore, we observe that both $\beta$-arrestin- 1 and $\beta$-arrestin-2 interacted with ATG7, individually, which can be enhanced by HG treatment. Therefore, we propose that the enhanced interaction between $\beta$-arrestin-1/2 and ATG7 may block ATG7 to freely access and activate glycine residue of ATG12, thereby reducing ATG12-ATG5 conjugation and suppressing autophagy (Figure 8). Further studies are needed to clarify the precise mechanisms bridging autophagy and $\beta$-arrestin-1/2 through mediating the enzymatic activity of ATG7.

It should be noted that, although we focus on podocyte function regulated by $\beta$-arrestins in $\mathrm{DN}$, the upregulation was also observed in both proximal tubules and distal tubules in the kidney from diabetic mice and patients. Recent studies have found that tubular epithelial cells such as HK-2 and LLC-PK1 (porcine) subjected to $\mathrm{HG}$ exhibited inhibition of autophagy and altered expression of mitophagic proteins. ${ }^{25}$ Therefore, it is necessary to further clarify whether autophagy in tubular epithelial cells shares the same mechanisms as podocytes regulated by $\beta$-arrestins in DN.

In addition, although in this study we demonstrated that $\beta$-arrestin- 1 and $\beta$-arrestin- 2 suppress podocyte autophagy by negative regulation of ATG12-ATG5 conjugation, thereby resulting in podocyte injury, we cannot exclude that other mechanisms are also involved in the regulation of podocyte function. Wnt/ $\beta$-catenin signaling cascade exhibits a pivotal function in the progression of DN and other proteinuric kidney diseases. ${ }^{26-29}$ Our preliminary studies have also found that phosphoprotein disheveled 2 (DVL-2), an integral part of Wnt signaling, ${ }^{30}$ is associated with and upregulated by $\beta$-arrestins in podocytes with HG treatment. This association between $\beta$-arrestins and DVL-2 inhibits $\beta$-catenin degradation, thereby activating $\mathrm{Wnt} / \beta$-catenin signaling (unpublished data). Considering recent studies indicating that $\mathrm{Wnt} / \beta$-catenin signaling has a central role in mediating podocyte dysfunction and proteinuria by controlling the rennin-angiotensin system and Snail signaling, ${ }^{31}$ it is possible that $\beta$-arrestin-DVL-2$\mathrm{Wnt} / \beta$-catenin axis besides autophagy inhibition may promote podocyte injuries in DN. Moreover, GPCRs have attracted considerable attention in DN. Numerous GPCRs such as receptors for angiotensin II, endothelin, thromboxane and E-series prostaglandins have been identified in podocytes and implicated in the progression of glomerular diseases. GPCRactivated signaling pathways are associated with the activation of phospholipase $\mathrm{C}$ through Gaq. Wang et al. ${ }^{32}$ 
demonstrated that the Gaq-coupled signaling pathways in glomerular podocytes promoted renal injury. In addition, Ga 12 couples to mumerous GPCRs and regulates multiple epithelial responses such as apoptosis, permeability and the actin cytoskeleton. Recent studies found that $\mathrm{Ga} 12$ activation in podocytes led to proteinuria and glomerulosclerosis via regulating collagen $\mathrm{IV}^{33}$ Considering that $\beta$-arrestins are originally identified as negative adaptors of GPCRs and a very recent study reveals that $\beta$-arrestin- 1 drives endothelin-1mediated podocyte activation and sustains renal injury in adriamycin-induced nephropathy, ${ }^{34}$ it is very possible that $\beta$-arrestins may also regulate GPCR signaling in podocytes by regulation of their desensitization and internalization, thereby leading to podocyte injury. However, how $\beta$-arrestins regulate GPCR in podocyte pathology under disease conditions remain to be investigated.

In conclusion, this study for the first time explores the role of $\beta$-arrestins in DN and provides direct evidence that $\beta$-arrestin1 and $\beta$-arrestin- 2 share common mechanisms to mediate podocyte autophagy by negative regulation of ATG12-ATG5 conjugation, indicating that $\beta$-arrestins are critical components of multiple signal transduction pathways that link renal injury to reduced autophagy in DN. Modulation of these pathways may be an innovative therapeutic strategy for treating patients with DN.

\begin{abstract}
Materials and Methods
Animal studies. The Arrb1- and Arrb2-deficient mice $\left(\right.$ Arrb1 $^{-/}$and Arrb2 $\left.^{-1-}\right)$ were originally produced by Dr. Lefkowitz's laboratory at Duke University (Durham, NC, USA) as described ${ }^{35,36}$ which have been crossed back to C57BL/6 mice for $>16$ generation. After transferring to Shandong University, these mice are crossed with C57BL/6 genetic mice for $>10$ generations. Mouse models of diabetes were developed by induction of streptozotocin (STZ) into 10-week-old male Arrb1 $1^{-/-}$, Arrb2 $^{-/-}$or WT C57BL/6 mice. Because mice with a C57BL/6 background do not develop lesions of DN readily after the induction of diabetes by STZ, Unx was performed to hasten the development of DN following previous studies. ${ }^{37}$ Briefly, after a 1-week recovery period from Unx, diabetes was induced by intraperitoneal injection of STZ (100 mg/kg body weight for 3 consecutive days). An equivalent amount of sodium citrate buffer alone was used as a vehicle control. After mice were injected with an intraperitoneal injection of STZ, blood glucose levels were monitored $48 \mathrm{~h}$ later and periodically thereafter (LifeScan One Touch glucometer, Johnson \& Johnson, Milpitas, CA, USA) by mice-tailed blood sampling. Mice with blood glucose levels $>15.0 \mathrm{mmol} / \mathrm{l}$ were considered as diabetic. All mice had unrestricted access to food/water and were maintained for 12 weeks in accordance with Institutional Animal Care and Use Committee procedures of Shandong University. At the end of the study, urine was collected for $24 \mathrm{~h}$ in a metabolic cage and urinary albumin excretion was measured using a mouse albumin ELISA Quantitation Kit (Bethyl Laboratories, Montgomery, TX, USA). Simultaneously, mice were killed under ketamine anesthesia. The fixed kidneys were paraffin embedded, and the sections were prepared and stained with periodic acid-Schiff stain. Renal tissue was homogenized for immunoblotting and mRNA analysis. The investigation conforms to the US National Institutes of Health Guide for the Care and Use of Laboratory Animals.
\end{abstract}

$\boldsymbol{d} \boldsymbol{b} / \boldsymbol{d} \boldsymbol{b}$ mice. Twelve-week-old male type 2 diabetic $d b / d b$ mice and genetic control $d b /+$ mice were obtained from the Jackson laboratory (Bar Harbor, ME, USA) as described. ${ }^{7}$

Human renal biopsy samples. Renal biopsies had been performed as part of routine clinical diagnostic investigation and collected as described in Supplementary Table S2. Among them, DM-NN were selected from patients who underwent nephrectomy for solitary renal cell carcinoma and had a concomitant diagnosis of type 2 diabetes. Histological examinations and biochemical analysis (urine $A C R<30 \mathrm{mg} / \mathrm{g}$ ) revealed no features of DN or other renal disease except for the solitary renal cell carcinoma. The samples of renal biopsies were obtained from the Department of Pathology, Shandong University School of Medicine, Jinan, China and the Department of Nephrology, Union Hospital, Tongji Medical College, Huazhong University of Science and Technology, Wuhan, China. Control samples were obtained from the healthy kidney poles of individuals who underwent tumor nephrectomies without diabetes or renal disease (Supplementary Table S2). The investigations were conducted in accordance with the principles of the Declaration of Helsinki and were approved by the Research Ethics Committee of Shandong University after informed consent was obtained from the patients.

Transmission electron microscopy. Electron microscopic sample handling and detection were performed by the electron microscopic core lab of Shandong University as described. ${ }^{38,39}$ TEM images were analyzed using Image J (National Institutes of Health, NIH, Bethesda, MD, USA) and analysis was based on previous studies. ${ }^{40,41}$ The GBM thickness, foot process width and the number of foot processes per $\mu \mathrm{m}$ of GBM were calculated using a curvimeter (SAKURAI CO., LTD, Tokyo, Japan) as described. ${ }^{42}$ Five glomeruli were randomly selected from each mouse and 10 electron micrographs were taken in each glomerulus.

Cell culture and treatments. Conditionally immortalized human podocytes were originally provided by Dr. Saleem MA from University of Bristol at Bristol, UK and were cultured in RPMI 1640 medium containing $11.0 \mathrm{mmol} / \mathrm{l}$ glucose as described. ${ }^{43,44}$ Different stimuli were used in this study: (1) HG and mannitol was used as the osmolarity control; to study the effect of $\mathrm{HG}$, glucose at a final concentration of 20 or $40 \mathrm{mmol} / /$ (additional 9.0 or $29.0 \mathrm{mmol} / \mathrm{l}$ glucose was added in the medium for podocytes) were employed in this study and mannitol $(29.0 \mathrm{mmo} / \mathrm{l}$ mannitol was added in the medium for podocytes); (2) AGE (50-200 $\mu \mathrm{g} / \mathrm{ml})$; and (3) TGF- $\beta 1(2-8 \mathrm{ng} / \mathrm{ml})$. In addition, chloroquine $(30 \mu \mathrm{mo} / /)$ or bafilomycin $\mathrm{A} 1$ $(50 \mathrm{nmol} / /)$ was used to pretreat cells.

In situ hybridization. Formalin-fixed, paraffin-embedded kidney tissues were sectioned (6- $\mu$ m thickness) and assayed for Arrb1 and Arrb2 RNA expression by ISH as described previously. ${ }^{45,46,47}$ Arrb2 was detected by the Arrb2 mRNA ISH Kit with digoxigenin (DIG)-labeled probe (Boster Biological Technology Co., Wuhan, China) according to the manufacturer's protocols. The DIG-labeled probe for Arrb1 (5'-CATTGGTGTCAAGCTCTATGAGATTGGTGTCTACTGGAGTCTCGC-3') was designed and synthesized by Shanghai Sunny Biotech Co., Ltd (Shanghai, China). Scramble probes (5'-CATTGGTGTCCCAGACTATGTTCAGGGTGTCTACT CATCATCTCGC-3') were used as negative controls.

RNA interference and overexpression of $\beta$-arrestins. Small interfering RNA (siRNA) to atg3 (5'-CACUUCCAGUGCCUUUCCC- $\left.3^{\prime}\right)$ was synthesized by BioSune. (Jinan, China). siRNA to Arrb1 or Arrb2 (siRNA- $\beta$-arrestin-1 or siRNA$\beta$-arrestin-2) was synthesized and constructed into PRNAT-U6.1/Neo to get shRNA$\beta$-arrestin-2 or shRNA- $\beta$-arrestin-2 by Biomics Biotechnologies Co., Ltd. (Nantong, China). The DNA target sequence for shRNA- $\beta$-arrestin- 1 is $5^{\prime}$-AAAGCCTTCT GTGCTGAGAAC- $3^{\prime}$ and the DNA target sequence for shRNA- $\beta$-arrestin-2 is $5^{\prime}$-AAGGACCGGAAAGTGTTCGTG-3'. In these experiments, shRNA was transfected by Lipofectamine 2000 (Invitrogen, Gaithersburg, MD, USA) according to the manufacturer's instruction. For overexpressions of $\beta$-arrestin-1 and $\beta$-arrestin-2, podocytes were transfected with pCMV6- $\beta$-arrestin-1 or pCMV6- $\beta$-arrestin-2 plasmids by Lipofectamine 2000 as described. ${ }^{48}$

RNA extraction and real-time RT-PCR. Total RNA was isolated from mouse kidney or cells using TRIzol reagent (Invitrogen) as described previously. ${ }^{49}$ mRNA levels for target genes were analyzed by real-time quantitative RT-PCR using a Bio-Rad iCycler system (Bio-Rad, Hercules, CA, USA). The specific primers for target genes in this study are listed in Supplementary Table S3.

Western blotting and immunoprecipitation analyses. Total cellular lysate preparation and western blotting analysis were performed as described previously. ${ }^{50}$ Antibodies used in this study are summarized in Supplementary Table S4. To document the loading controls, the membrane was reprobed with a primary antibody against housekeeping protein GAPDH. For measurement of the interaction between target molecules, cellular lysates were analyzed by immunoprecipitation as described..$^{51}$

Immunofluorescence staining and confocal microscopy. Primary polyclonal antibodies $\beta$-arrestin-1 and $\beta$-arrestin-2 (1:100 dilution, ProteinTech 
Group, Chicago, IL, USA) were used for immunofluorescent staining, and images were obtained by a LSM780 laser scanning confocal microscope (ZEISS, Jena, Germany) system as described. ${ }^{52,37}$ To monitor the various stages of autophagy, the tandem GFP-RFP-LC3 adenovirus construct obtained from Hanbio Inc. (Shanghai, China) was used in this study. This tandem GFP-RFP-LC3 construct capitalizes on the $\mathrm{pH}$ difference between the acidic autolysosome and the neutral autophagosome and the $\mathrm{pH}$ sensitivity differences exhibited by GFP and RFP to monitor progression from the autophagosome to autolysosome. Briefly, to perform image-based analysis for autophagy, podocytes were infected with the tandem GFP-RFP-LC3 adenovirus for $24 \mathrm{~h}$, and then the cells were treated and imaged for GFP and RFP by using confocal fluorescence microscopy.

Statistics. Data are expressed as means \pm S.E.M. The significance of the differences in mean values between and within multiple groups was examined by one-way ANOVA followed by Duncan's multiple range test. $P<0.05$ was considered statistically significant.

\section{Conflict of Interest}

The authors declare no conflict of interest.

Acknowledgements. We acknowledge Miss Bao Chanchan at the Microscopy Characterization Platform, Shandong University for technical assistance in confocal microscopy. This work was funded by The National Science Fund for Distinguished Young Scholars to F Yi (81525005). This study was supported by the National 973 Basic Research Program of China (2012CB517700); The National Nature Science Foundation of China (81170772, 81371317, 81328006 and 81470958); Program for Changjiang Scholars and Innovative Research Team in University (PCSIRT, IRT13028); and the Fundamental Research Funds of Shandong University (2014JC027).

\section{Author contributions}

$\mathrm{JL}, \mathrm{JS}$ and $\mathrm{FY}$ designed the study, wrote the paper and approved its final version. JL, $\mathrm{QL}, \mathrm{XW}, \mathrm{YD}, \mathrm{ZW}$ and $\mathrm{YZ}$ contributed to the study design, acquisition of data, figure preparation and approved its final version. $\mathrm{XY}$ and NL contributed to animal studies, interpretation of data and approved the final version. $\mathrm{CZ}$ contributed to the clinical data analysis and description. FY is responsible for the integrity of the work as a whole.

1. Mizushima N, Komatsu M. Autophagy: renovation of cells and tissues. Cell 2011; 147 728-741

2. Takabatake $\mathrm{Y}$, Kimura T, Takahashi A, Isaka Y. Autophagy and the kidney: health and disease. Nephrol Dial Transplant 2014; 29: 1639-1647.

3. Huber TB, Edelstein CL, Hartleben B, Inoki K, Jiang M, Koya D et al. Emerging role of autophagy in kidney function, diseases and aging. Autophagy 2012; 8: 1009-1031.

4. Kume S, Thomas MC, Koya D. Nutrient sensing, autophagy, and diabetic nephropathy. Diabetes 2012; 61: 23-29.

5. Hartleben B, Godel M, Meyer-Schwesinger C, Liu S, Ulrich T, Kobler S et al. Autophagy influences glomerular disease susceptibility and maintains podocyte homeostasis in aging mice. J Clin Invest 2010; 120: 1084-1096.

6. Bechtel W, Helmstadter M, Balica J, Hartleben B, Kiefer B, Hrnjic F et al. Vps34 deficiency reveals the importance of endocytosis for podocyte homeostasis. J Am Soc Nephrol 2013; 24: $727-743$.

7. Wang X, Liu J, Zhen J, Zhang C, Wan Q, Liu G et al. Histone deacetylase 4 selectively contributes to podocyte injury in diabetic nephropathy. Kidney Int 2014; 86: 712-725.

8. Zhang MZ, Wang Y, Paueksakon P, Harris RC. Epidermal growth factor receptor inhibition slows progression of diabetic nephropathy in association with a decrease in endoplasmic reticulum stress and an increase in autophagy. Diabetes 2014; 63: 2063-2072.

9. Spiegel A. Cell signaling. beta-arrestin-not just for $G$ protein-coupled receptors. Science 2003; 301: 1338-1339.

10. Pierce KL, Lefkowitz RJ. Classical and new roles of beta-arrestins in the regulation of G-protein-coupled receptors. Nat Rev Neurosci 2001; 2: 727-733.

11. Quack I, Rump LC, Gerke P, Walther I, Vinke T, Vonend $O$ et al. Beta-arrestin2 mediates nephrin endocytosis and impairs slit diaphragm integrity. Proc Natl Acad Sci USA 2006; 103: 14110-14115.

12. Quack I, Woznowski M, Potthoff SA, Palmer R, Konigshausen E, Sivritas S et al. PKC alpha mediates beta-arrestin2-dependent nephrin endocytosis in hyperglycemia. $\mathrm{J}$ Biol Chem 2011; 286: 12959-12970.
13. Yuan K, Huang C, Fox J, Laturnus D, Carlson E, Zhang B et al. Autophagy plays an essential role in the clearance of Pseudomonas aeruginosa by alveolar macrophages. J Cell Sci2012; 125: $507-515$

14. Sun $\mathrm{T}$, Li X, Zhang $\mathrm{P}$, Chen WD, Zhang $\mathrm{HL}$, Li DD et al. Acetylation of Beclin 1 inhibits autophagosome maturation and promotes tumour growth. Nat Commun 2015; 6: 7215

15. Sharifi MN, Mowers EE, Drake LE, Macleod KF. Measuring autophagy in stressed cells. Methods Mol Biol 2015; 1292: 129-150.

16. Yi F, dos Santos EA, Xia M, Chen QZ, Li PL, Li N. Podocyte injury and glomerulosclerosis in hyperhomocysteinemic rats. Am J Nephrol 2007; 27: 262-268.

17. Mathieson PW. The podocyte as a target for therapies-new and old. Nat Rev Nephrol 2012 8: $52-56$.

18. Periyasamy-Thandavan S, Jiang M, Schoenlein P, Dong Z. Autophagy: molecular machinery, regulation, and implications for renal pathophysiology. Am J Physiol Renal 2009; 297: F244-F256.

19. Sun Q, Fan W, Chen K, Ding X, Chen S, Zhong Q. Identification of Barkor as a mammalian autophagy-specific factor for Beclin 1 and class III phosphatidylinositol 3-kinase. Proc Nat Acad Sci USA 2008; 105: 19211-19216.

20. Kang R, Zeh HJ, Lotze MT, Tang D. The Beclin 1 network regulates autophagy and apoptosis. Cell Death Differ 2011; 18: 571-580.

21. Wang $P, X u$ TY, Wei K, Guan YF, Wang X, Xu H et al. ARRB1/beta-arrestin-1 mediates neuroprotection through coordination of BECN1-dependent autophagy in cerebral ischemia. Autophagy 2014; 10: 1535-1548.

22. Walczak M, Martens S. Dissecting the role of the Atg12-Atg5-Atg16 complex during autophagosome formation. Autophagy 2013; 9: 424-425.

23. Kaushal GP. Autophagy protects proximal tubular cells from injury and apoptosis. Kidney Int 2012; 82: 1250-1253.

24. Taherbhoy AM, Tait SW, Kaiser SE, Williams AH, Deng A, Nourse A et al. Atg8 transfer from Atg7 to Atg3: a distinctive E1-E2 architecture and mechanism in the autophagy pathway. Mol Cell 2011; 44: 451-461.

25. Zhan M, Usman IM, Sun L, Kanwar YS. Disruption of renal tubular mitochondrial quality control by Myo-inositol oxygenase in diabetic kidney disease. J Am Soc Nephrol 2015; 26 : 1304-1321.

26. Lin CL, Wang JY, Huang YT, Kuo YH, Surendran K, Wang FS. Wnt/beta-catenin signaling modulates survival of high glucose-stressed mesangial cells. J Am Soc Nephrol 2006; 17: 2812-2820.

27. Zhou T, He X, Cheng R, Zhang B, Zhang RR, Chen Y et al. Implication of dysregulation of the canonical wingless-type MMTV integration site (WNT) pathway in diabetic nephropathy. Diabetologia 2012; 55: 255-266.

28. Clevers H, Nusse R. Wnt/beta-catenin signaling and disease. Cell 2012; 149: 1192-1205.

29. He W, Kang YS, Dai C, Liu Y. Blockade of Wnt/beta-catenin signaling by paricalcitol ameliorates proteinuria and kidney injury. J Am Soc Nephrol 2011; 22: 90-103.

30. Bryja V, Gradl D, Schambony A, Arenas E, Schulte G. Beta-arrestin is a necessary component of Wnt/beta-catenin signaling in vitro and in vivo. Proc Natl Acad Sci USA 2007 104: 6690-6695.

31. Zhou L, Liu Y. Wnt/beta-catenin signalling and podocyte dysfunction in proteinuric kidney disease. Nat Rev Nephrol 2015; 11: 535-545.

32. Wang L, Fields TA, Pazmino K, Dai Q, Burchette JL, Howell DN et al. Activation of Galpha q-coupled signaling pathways in glomerular podocytes promotes renal injury. J Am Soc Nephrol 2005; 16: 3611-3622.

33. Boucher I, Yu W, Beaudry S, Negoro H, Tran M, Pollak MR et al. Galpha12 activation in podocytes leads to cumulative changes in glomerular collagen expression, proteinuria and glomerulosclerosis. Lab Invest 2012; 92: 662-675.

34. Buelli S, Rosano L, Gagliardini E, Corna D, Longaretti L, Pezzotta A et al. beta-arrestin-1 drives endothelin-1-mediated podocyte activation and sustains renal injury. J Am Soc Nephrol 2014; 25: 523-533.

35. Hara MR, Kovacs JJ, Whalen EJ, Rajagopal S, Strachan RT, Grant W et al. A stress response pathway regulates DNA damage through beta2-adrenoreceptors and betaarrestin-1. Nature 2011; 477: 349-353.

36. Wang HM, Dong JH, Li Q, Hu Q, Ning SL, Zheng W et al. A stress response pathway in mice upregulates somatostatin level and transcription in pancreatic delta cells through Gs and beta-arrestin 1. Diabetologia 2014; 57: 1899-1910.

37. Lin M, Yiu WH, Wu HJ, Chan LY, Leung JC, Au WS et al. Toll-like receptor 4 promotes tubular inflammation in diabetic nephropathy. J Am Soc Nephrol 2012; 23: 86-102.

38. Du P, Fan B, Han H, Zhen J, Shang J, Wang X et al. NOD2 promotes renal injury by exacerbating inflammation and podocyte insulin resistance in diabetic nephropathy. Kidney Int 2013; 84: 265-276.

39. Zhang S, Zhang Y, Wei X, Zhen J, Wang Z, Li M et al. Expression and regulation of a novel identified TNFAIP8 family is associated with diabetic nephropathy. Biochim Biophys Acta 2010; 1802: 1078-1086

40. Whaley-Connell A, Habibi J, Nistala R, Cooper SA, Karuparthi PR, Hayden MR et al. Attenuation of NADPH oxidase activation and glomerular filtration barrier remodeling with statin treatment. Hypertension 2008; 51: 474-480.

41. Lenoir O, Jasiek M, Henique C, Guyonnet L, Hartleben B, Bork T et al. Endothelial cell and podocyte autophagy synergistically protect from diabetes-induced glomerulosclerosis. Autophagy 2015; 11: 1130-1145. 
42. Kojima K, Nosaka H, Kishimoto Y, Nishiyama Y, Fukuda S, Shimada M et al. Defective glycosylation of alpha-dystroglycan contributes to podocyte flattening. Kidney Int 2011; 79: 311-316.

43. Coward RJ, Welsh Gl, Yang J, Tasman C, Lennon R, Koziell A et al. The human glomerular podocyte is a novel target for insulin action. Diabetes 2005; 54: 3095-3102.

44. Saleem MA, O'Hare MJ, Reiser J, Coward RJ, Inward CD, Farren T et al. A conditionally immortalized human podocyte cell line demonstrating nephrin and podocin expression. J Am Soc Nephrol 2002; 13: 630-638.

45. Canaud G, Dejucq-Rainsford N, Avettand-Fenoel V, Viard JP, Anglicheau D, Bienaime F et al. The kidney as a reservoir for HIV-1 after renal transplantation. J Am Soc Nephrol 2014; 25: 407-419.

46. Ichii O, Otsuka S, Sasaki N, Namiki Y, Hashimoto Y, Kon Y. Altered expression of microRNA miR-146a correlates with the development of chronic renal inflammation. Kidney Int 2012; 81: 280-292.

47. McArthur K, Feng B, Wu Y, Chen S, Chakrabarti S. MicroRNA-200b regulates vascular endothelial growth factor-mediated alterations in diabetic retinopathy. Diabetes 2011; 60 1314-1323.

48. He M, Zhang B, Wei X, Wang Z, Fan B, Du P et al. HDAC4/5-HMGB1 signalling mediated by NADPH oxidase activity contributes to cerebral ischaemia/reperfusion injury. J Cell Mol Med 2013; 17: 531-542

49. Wang Z, Wei X, Zhang $Y$, Ma X, Li B, Zhang $S$ et al. NADPH oxidase-derived ROS contributes to upregulation of TRPC6 expression in puromycin aminonucleoside-induced podocyte injury. Cell Physiol Biochem 2009; 24: 619-626.
50. Yi F, Zhang AY, Janscha JL, Li PL, Zou AP. Homocysteine activates NADH/NADPH oxidase through ceramide-stimulated Rac GTPase activity in rat mesangial cells. Kidney Int 2004; 66 : 1977-1987.

51. Stronach EA, Alfraidi A, Rama N, Datler C, Studd JB, Agarwal R et al. HDAC4-regulated STAT1 activation mediates platinum resistance in ovarian cancer. Cancer Res 2011; 71: 4412-4422.

52. Han H, Wang Y, Li X, Wang PA, Wei X, Liang W et al. Novel role of NOD2 in mediating Ca2+ signaling: evidence from NOD2-regulated podocyte TRPC6 channels in hyperhomocysteinemia. Hypertension 2013; 62: 506-511.

(i) Cell Death and Disease is an open-access journal published by Nature Publishing Group. This work is licensed under a Creative Commons Attribution 4.0 International License. The images or other third party material in this article are included in the article's Creative Commons license, unless indicated otherwise in the credit line; if the material is not included under the Creative Commons license, users will need to obtain permission from the license holder to reproduce the material. To view a copy of this license, visit http://creativecommons.org/licenses/by/4.0/

Supplementary Information accompanies this paper on Cell Death and Disease website (http://www.nature.com/cddis) 